\title{
Understanding Pattern Hair Loss-Hair Biology Impacted by Genes, Androgens, Prostaglandins and Epigenetic Factors
}

\author{
Ralph M. Trüeb ${ }^{1}$ \\ ${ }^{1}$ Center for Dermatology and Hair Diseases Professor Trüeb, \\ Switzerland \\ Indian J Plast Surg 2021;54:385-392.
}

\author{
Address for correspondence Ralph M. Trüeb, MD, Center for \\ Dermatology and Hair Diseases Professor Trüeb, Bahnhofplatz 1A, CH- \\ 8304 Wallisellen, Switzerland \\ (e-mail: r.trueeb@derma-haarcenter.ch).
}

\begin{abstract}
Keywords

- Pattern hair loss

- Oral finasteride

- Topical minoxidil

- Minoxidil sulfate

- Topical finasteride

- Oral minoxidil

- Oxidative Stress

- Microinflammation

Pattern hair loss (PHL) is the most frequent cause of hair loss in men and women, accounting for $65 \%$ of consultations in a hair referral center. PHL is understood to represent a hereditary, age-dependent progressive thinning of the scalp hair, which follows distinct clinical patterns with notable differences depending on sex and age of onset. Clinical and investigative advances have helped us to understand some of the pathogenic steps, leading to PHL. Besides genetic factors and peculiarities of androgen metabolism, additional pathogenic factors that are suspected include microbiomata, oxidative stress, and microinflammation. While further suspects are likely to be exposed, individual diversity of causal agents, as well as of the sequence of events, or combined factors, must be kept in mind. A large number of therapeutic molecules claimed to be active and patented in this field, and their limited efficacy in offering a definitive cure of PHL confirm the complexity of PHL. The aim of therapy is to retard progression of hair thinning and increase hair coverage of the scalp. As yet, two FDAapproved drugs are available for this purpose, oral finasteride, and topical solution of minoxidil. Variations in posology and formulation allow for an enhancement of patient comfort and treatment efficacy. Antiandrogen treatments in women with normal androgen levels have questionable efficacy while having health risks.
\end{abstract}

\section{Pattern Hair Loss}

Pattern hair loss (PHL) is the most frequent cause of alopecia, with a frequency of $65 \%$ of 12,880 patients consulting our center for hair loss over a period of 10 years. PHL affects at least $50 \%$ of men by the age of 50 years, and up to $70 \%$ of all males in later life, ${ }^{1}$ while estimates of its prevalence in women have varied widely. Recent studies claim that $6 \%$ of women aged under 50 years are affected, increasing to a proportion of 30 to $40 \%$ of women aged 70 years and over. ${ }^{2}$
PHL is understood to represent a hereditary, androgendependent, progressive thinning of the scalp hair with age, which follows defined patterns, with notable differences between sexes with respect to the frequency of the condition, the age of onset, and the clinical presentation.

While male PHL (MPHL) is characterized by bitemporal recession of the hair and balding vertex, female PHL (FPHL) is set apart by its diffuse thinning of the crown and intact frontal hairline. As much as $13 \%$ of premenopausal women published online

December 14, 2021
DOI https://doi.org/

$10.1055 / \mathrm{s}-0041-1739252$. ISSN 0970-0358. (c) 2021. Association of Plastic Surgeons of India. All rights reserved. This is an open access article published by Thieme under the terms of the Creative Commons Attribution-NonDerivative-NonCommercial-License, permitting copying and reproduction so long as the original work is given appropriate credit. Contents may not be used for commercial purposes, or adapted, remixed, transformed or built upon. (https://creativecommons.org/ licenses/by-nc-nd/4.0/)

Thieme Medical and Scientific Publishers Pvt. Ltd., A-12, 2nd Floor, Sector 2, Noida-201301 UP, India 
and $37 \%$ of postmenopausal women present with the male pattern. ${ }^{3}$ Before puberty, PHL presents in both sexes with the female pattern. ${ }^{4}$ A more diffuse pattern of hair loss over the vertex and frontal hair line, resembling female pattern hair loss, may occur in men, ${ }^{5}$ and it seems to be more common in Asians

In relation to hair cycling, PHL is pathodynamically characterized by progressive shortening of the duration of anagen within successive hair cycles, leading to a decreased proportion of hair in anagen, and progressive follicular miniaturization with conversion of terminal to vellus-like follicles. ${ }^{6}$ The result is increased shedding of short-lived telogen hairs, while the affected hair follicles produce shorter, finer hairs that eventually cover the scalp poorly.

Since PHL involves a process of premature termination of anagen associated with premature entry into catagen, it is critically important to dissect the molecular controls of the anagen-catagen transformation of the hair cycle. ${ }^{7}$ Catagen has been suggested to occur as a consequence of decreased expression of anagen-maintaining factors such as insulinlike growth factor 1 (IGF-1), basic fibroblast growth factor (bFGF), vascular endothelial growth factor (VEGF), and increased expression of cytokines promoting apoptosis such as transforming growth factor $\beta 1$ (TGFbeta 1 ), interleukin- $1 \alpha$ (IL-1 $\alpha$ ), and tumor necrosis factor $\alpha$ (TNFalpha).

Response to androgens seems to be intrinsic to the individual hair follicle: not only does the response vary from stimulation to inhibition of hair growth, depending on the body site, but androgen sensitivity also varies within individual areas, that is, regression in PHL occurs in a patterned manner, with peculiarities of local androgen metabolism playing a role. ${ }^{8}$

Since extrinsic hair growth-modulatory factors such as androgens ${ }^{9}$ apparently operate via the dermal papilla, research is currently focused on identifying androgen-regulated factors derived from dermal papilla cells. Of the several factors that were suggested to play a role in hair growth, IGF1 was reported as altered in vitro by androgens, ${ }^{10}$ and stem cell factor (SCF) was found to be produced in higher amounts by androgen-dependent beard cells than control nonbalding scalp cells, presumably also in response to androgens. ${ }^{11}$

Among the conditions with age-dependent loss of hair, PHL represents the most important cause, while the progerias (Hutchinson-Gilford and Werner's syndrome) and other rare conditions associated with premature PHL such as the Laron syndrome and myotonic dystrophy (Curshmann-Steinert) provide insights into the roles of DNA instability and repair mechanisms, telomeres, mitochondrial function, human growth hormone (HGH), and IGF-1 for the growth and aging of hair.

At the other end of the life span, senescent alopecia has been defined as nonandrogen-dependent hair thinning found in those over 50 years of age. ${ }^{12}$ Much like PHL, it involves a progressive decline in the number of anagen follicles and hair diameter. However, data comparing gene expression profiles in PHL and senescent alopecia using microarray analysis have demonstrated differences, suggesting that, in fact, they represent different entities. In PHL, genes required for anagen onset (Wnt- $\beta$-catenin, TGF- $\alpha$, TGF$\beta$, Stat-3, Stat-1), epithelial signal to dermal papilla (PPARd, IGF-1), hair shaft differentiation (Notch, Msx2, KRTs, KAPs), and anagen maintenance (Msx2, Activin, IGF-1) were downregulated, and genes for catagen (BDNF, BMP2, BMP7, VDR, IL-1, ER) and telogen induction and maintenance (VDR, RAR) were upregulated. In senescent alopecia, genes involved in epithelial signal to dermal papilla (FGF5), actin cytoskeleton (DST, ACTN2, TNNI3, PARVB) and mitochondrial function (JAK2, PRKD3, AK2, TRAP1, TRIO, ATP12A, MLL4, STK22B) were downregulated, while oxidative stress and inflammatory response genes were upregulated. ${ }^{13}$

\section{Genetics}

The genetic involvement is pronounced in PHL, and the importance of genes concurs with marked racial differences in the prevalence of PHL; non-Caucasians often exhibit significantly less balding. While major progress has been done in the understanding of androgen metabolism, the genetic predisposition to PHL remains poorly understood. A very high frequency of PHL has complicated attempts to establish a mode of inheritance. Moreover, it is not clear whether PHL is genetically homogeneous; some authorities suggest that FPHL is not the female counterpart of MPHL and not androgen dependent. ${ }^{14}$ The genes for type 1 and type 2 $5 \alpha$-reductase were shown not to be associated with the inheritance of PHL, ${ }^{15}$ while polymorphisms of the androgen receptor (AR) gene have been shown to be associated with MPHL. ${ }^{16}$ However, the AR gene is located on the X chromosome and does not explain the relatively strong concordance of the degree of baldness in fathers and sons. No specific gene has been identified so far, although single gene mutations such as abnormality of the AR might be necessary but not sufficient for the phenotype. ${ }^{16}$ In fact, we are dealing with a polygenic inheritance dependent on a combination of mutations, for example, in or around the AR gene affecting the expression of the AR and other genes controlling androgen metabolism. Interactions between such genes might account for the tissue-specific and developmental stage-specific expression of the AR, which is necessary to explain the characteristic anatomic and temporal patterns of PHL. Other genes relevant to androgens, including those on the Y chromosome, might also be examined. ${ }^{16}$

\section{Role of Sexual Hormones}

Of the various hormones that affect hair growth, the most studied are the androgens. ${ }^{17}$ Since Aristotle first noted that maleness and sexual maturity were required for balding, Hamilton's observations on men deprived of testicular androgens by castration established beyond doubt that androgens were prerequisites for development of MPHL. ${ }^{18}$

Androgen metabolism comprises glandular and extraglandular production, transport, target cell metabolism, and cellular response. While androgen biology in the adrenals and gonads, and the influence of the pituitary axis, go beyond the scope of this review, androgen metabolism 
within the skin, as it relates to the hair, is the focus ${ }^{17}$ : the androgen metabolism pathway begins with pregnenolone, a 21 carbon steroid substrate, converted from cholesterol. Following $\alpha$-hydroxylation at the $\mathrm{C}-17$ position, the action of the enzyme C17-20 lyase cleaves distal carbon moieties, leaving a C19 carbon steroid with a C-17 ketone in the distal ring. These 17-ketosteroids make up a group of weak androgens such as dehydroepiandrosterone (DHEA), defined by a low affinity for the AR. These weak androgens, however, can be enzymatically converted to more potent androgens with greater affinity for the AR such as testosterone $(T)$. $T$ is the major circulating androgen. In women, systemic levels of $T$ are low compared with men, but the more abundant weak androgens serve as a source of precursors for potent androgens, which provide the physiologic or pathophysiologic androgen activity. Only a small fraction of androgens exists as free steroids in the circulation, with an equilibrium between free hormones and protein-bound androgens. The most important protein for androgen binding is sex hormone-binding globulin (SHBG). Normally $70 \%$ of $\mathrm{T}$ is bound to SHBG and $19 \%$ to albumin. The remainder is circulating unbound. In most target organs, $\mathrm{T}$ can be metabolized to dihydrotestosterone (DHT) by the enzyme steroid $5 \alpha$-reductase (5AR). Based on its affinity for the AR, DHT is 5-fold more potent than T. DHT is implicated in the pathogenesis of several disorders, including benign prostatic hyperplasia, prostate cancer, hirsutism, and acne vulgaris, besides PHL.

The skin and pilosebaceous unit are enzymatically equipped for local metabolism and conversion of sex steroids ${ }^{17}$ : the skin is capable of synthesizing active androgens from the systemic precursor DHEA-sulfate (DHEA-S). The first step is the desulfation of DHEA-S by the enzyme steroid sulfatase (STS). The principal pathways involved in conversion of weak androgens like DHEA to more potent androgens are through activity of the enzymes $3 \beta$-hydroxysteroid

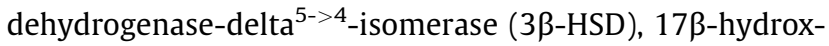
ysteroid dehydrogenase (17 $\beta-H S D)$, and 5AR. Once formed, potent androgens, such as T and DHT, can be removed by conversion back to the weaker 17-ketosteroids, or are metabolized via other enzymatic pathways, including aromatase, which convert androgens to estrogens, and $3 \alpha-$ hydroxysteroid dehydrogenase to form androsterone and androstanediol. The latter can be glucuronidated to form androgen conjugates that are more rapidly cleared from the circulation.

Remarkably, some target tissues, including the hair follicle, show enhanced androgen metabolism. The activity of enzymes involved in androgen metabolism within the skin has been studied in a variety of tissue preparations: the sebaceous glands in balding skin have been shown to express increased $3 \beta$-HSD activity when compared with nonbalding scalp areas. ${ }^{19}$ Very early, it was shown that plucked human hair follicles or hair follicles from balding stump-tailed macaques express considerable $17 \beta-H S D$ activity. ${ }^{20}$ In a study of plucked hair follicles from young adults not yet expressing AGA but with a strong family history of baldness, two populations were found, one with high $17 \beta$-HSD activity and one with low-enzyme activity. ${ }^{21}$ The study suggested that low-enzyme activity may be related to lesser degrees of balding. On the other hand, both men and women with PHL were shown to have higher levels of SAR enzyme activity in frontal follicles than in their own occipital follicles, whereas higher levels of aromatase were found in their occipital follicles. ${ }^{8}$

Since STS converts DHEA-S to DHEA that is eventually metabolized to the more potent androgens in the periphery, and elevated plasma levels of DHEA-S and DHEA have been reported to correlate with balding in young men, the hypothesis was advanced that men with genetic STS deficiency (X-linked recessive ichthyosis, XRI) do not or only minimally develop PHL. ${ }^{22}$ We conducted a survey of patients with XRI showing that this is not the case, since these men also showed advanced PHL. ${ }^{23}$

Eventually, the report of an unusual form of incomplete male pseudohermaphroditism, due to a genetic deficiency of the type 2 steroid 5AR by Imperato-McGinley et al, ${ }^{24}$ implicated DHT as the principal mediator of androgen-dependent hair loss: affected men, who are homozygous for mutation of the gene, do not develop PHL.

Finally, the absence of balding in individuals with the androgen-insensitivity syndrome (testicular feminization) and who lack functional AR has been put forth as evidence for the need of AR for PHL to occur ${ }^{25}$ and rational basis for antiandrogen therapy.

All steroid hormones act by diffusing through the plasma membrane into the target cell and binding to specific intracellular receptors. The hormone-receptor complex undergoes conformational changes, exposing DNA-binding sites, and then binds to specific hormone response elements in the DNA, promoting the expression of specific hormone-regulated genes. Ultimately, the AR is believed to be responsible for determining the sensitivity of cells to androgens. However, FPHL does develop in patients with the androgen-insensitivity syndrome, ${ }^{26}$ suggesting, together with the observation of PHL in prepubertal children ${ }^{4}$ and in patients with hypogonadism, that mechanisms other than direct androgen action contribute to FPHL, ${ }^{14}$ and antiandrogen therapy is not effective, as shown by Vexiau et al for the antiandrogen, cyproterone acetate (CPA), in women with normal serum androgen levels. ${ }^{27}$

Consistent with the role of estrogen on hair growth in women is that aromatase inhibitors for the treatment of breast cancer may lead to PHL-like alopecia in women. ${ }^{28}$

\section{Prostaglandins}

Garza et al originally showed that both prostaglandin $\mathrm{D}(2)$ synthase (PTGDS) activity and prostaglandin D(2) PGD (2) levels are elevated in bald scalp compared with the haired scalp of men with AGA. In mice, PTGDS and PGD(2) levels increase immediately preceding the regression phase during normal follicle cycling, suggesting an inhibitory effect on hair growth. The authors also showed that $\operatorname{PGD}(2)$ inhibits hair growth in explanted human hair follicles and when applied topically to mice. Hair growth inhibition requires the $\operatorname{PGD}(2)$ receptor $G$ protein (heterotrimeric guanine nucleotide)- 
coupled receptor 44 (GPR44) but not the PGD (2) receptor 1 (PTGDR). Furthermore, they found that a transgenic mouse, K14-PTGS2, which targets prostaglandin-endoperoxide synthase 2 expression to the skin, demonstrates elevated levels of PGD(2) in the skin and develops alopecia, follicular miniaturization, and sebaceous gland hyperplasia, which are all hallmarks of human AGA. According to the authors, these results define $\operatorname{PGD}(2)$ as an inhibitor of hair growth in AGA and suggest the PGD(2)-GPR44 pathway as a potential target for treatment. ${ }^{29}$

Chovarda et al further investigated the role of the four major prostaglandins (PGs) (PGE2, PGD2, PGF2 $\alpha$, and PI2 or prostacyclin) in the pathogenesis of AGA in males and females. They studied human scalp biopsies obtained from 30 adults (17 males and 13 females) suffering from AGA and from 30 healthy adults ( 18 males and 12 females). In each subject, two skin biopsies were performed, one from the frontal bald area and another one from the occipital area with normal hair growth. In the control group, biopsies were taken from both areas too. In each sample, all four PGs were measured. In the AGA group, PGD2 and prostacyclin or PGI2 levels were increased in bald compared with haired scalp. On the contrary, measurements of PGE2 and PGF2 $\alpha$ showed that they were diminished in bald areas compared with haired areas. PG levels in healthy samples were comparable to the haired areas. These results support that there is a fine balance between the four major PGs, PGE2, PGD2, PGF2 $\alpha$, and PI2 or prostacyclin in scalp hair follicles, and its maintenance may be crucial for hair growth. ${ }^{30}$

Bimatoprost and latanoprost are structural analogues of PG F2 $\alpha$ (PGF2 $\alpha$ ), originally used as a medication to treat glaucoma, which have been showed to enhance eyelash growth. They are currently being investigated for their hair growth-promoting potential in PHL. ${ }^{31}$

\section{Role of Follicular Microinflammation}

The limited success rate of treatment of PHL with hair growth promoters or modulators of androgen metabolism means that further pathogenic pathways must be taken into account. The implication of microscopic follicular inflammation in the pathogenesis of PHL has emerged from several studies. ${ }^{32,33}$ The term microinflammation was proposed by Mahé et al, because the process involves a slow, subtle, and indolent course, in contrast to the inflammatory and destructive process in the classical inflammatory scarring alopecias. ${ }^{34}$ Morphometric studies in patients with MPHL treated with minoxidil showed that $55 \%$ of those with microinflammation had regrowth in response to treatment, in comparison to $77 \%$ in those patients without inflammation and fibrosis. ${ }^{33}$ Finally, the more recently described fibrosing alopecia in a pattern distribution, ${ }^{35}$ a condition in which patients with PHL have additional clinical and histological features of inflammation and fibrosis limited to the area of PHL, provides the respective evidence.

An important question is how the inflammation is generated around the individual hair follicle. Inflammation is regarded a multistep process, which may start from a primary event. The observation of a perifollicular infiltrate in the upper follicle near the infundibulum suggests that the primary causal event for the triggering of inflammation might occur near the infundibulum. ${ }^{35}$ On the basis of this localization and the microbial colonization of the follicular infundibulum with Propionibacterium sp., Staphylococcus sp., Malassezia sp., or other members of the transient flora (microbiome), one could speculate that microbial toxins or antigens could be involved in the generation of the inflammatory response. In fact, the scalp is characterized by a usually high density of terminal hair growth with numerous sebaceous glands, which contribute to a specific microenvironment that is rich in microbes. Sebaceous areas have greater species richness than dry ones, with implications both for skin physiology and pathologic conditions. ${ }^{36}$ The production of porphyrins by Propionibacterium sp. in the pilosebaceous duct has also been considered to be a possible cofactor of this initial proinflammatory stress. Alternatively, keratinocytes themselves may respond to chemical stress from irritants, pollutants, and ultraviolet (UV) irradiation by producing radical oxygen species and nitric oxide and by releasing intracellularly stored IL- $1 \alpha^{34}$ This proinflammatory cytokine by itself has been shown to inhibit the growth of isolated hair follicles in culture. ${ }^{37}$ Moreover, adjacent keratinocytes, which express receptors for IL-1, start to engage in the transcription of IL-1 responsive genes: mRNA coding for IL-1 $\beta$, TNFbeta, and IL- $1 \alpha$, and for specific chemokine genes, such as IL-8, and monocyte chemoattractant protein-1 (MCP-1) and MCP-3, themselves mediators for the recruitment of neutrophils and macrophages, they have been shown to be upregulated in the epithelial compartment of the human hair follicle. Besides, adjacent fibroblasts are also fully equipped to respond to such a proinflammatory signal. The upregulation of adhesion molecules for bloodborne cells in the capillary endothelia, together with the chemokine gradient, drive the transendothelial migration of inflammatory cells, which include neutrophils through the action of IL-8, T-cells and Langerhans cells at least in part through the action of MCP-1. After processing of localized antigen, Langerhans cells, or alternatively keratinocytes, which may also have antigen presenting capabilities, could then present antigen to newly infiltrating T-lymphocytes and induce T-cell proliferation. The antigens are selectively destroyed by infiltrating macrophages or natural killer cells. ${ }^{34}$ On the occasion that the causal agents persist, sustained inflammation is the result, together with connective tissue remodeling, where collagenases such as matrix metalloproteinase (also transcriptionally driven by proinflammatory cytokines) play an active role. ${ }^{34}$

Eventually, permanent alopecia is the result of irreversible damage to the putative site of follicular stem cells in the bulge area of the outer-root sheath in the superficial portion of the hair follicle. ${ }^{38}$

\section{Role of Oxidative Stress}

Experimental evidence supports the hypothesis that oxidative stress plays a major role in the aging process. Harman 
et $\mathrm{al}^{39}$ originally proposed the free radical theory of aging: free radicals are highly reactive molecules with unpaired electrons that can directly damage various cellular structural membranes, lipids, proteins, and DNA. Keeping in mind the analogy of Harman's original free radical theory of aging, Arck et al proposed a free radical theory of greying: ${ }^{40,41}$ the extraordinary melanogenic activity of pigmented bulbar melanocytes, continuing for up to 10 years in some hair follicles, is likely to generate large amounts of reactive oxygen species via the hydroxylation of tyrosine and the oxidation of DOPA to melanin. If not adequately removed by an efficient antioxidant system, an accumulation of these reactive oxidative species will generate significant oxidative stress.

The condition of the hair fiber must be viewed as the result of a combination of preemergent and postemergent factors. ${ }^{42}$ Sources of oxidative stress with impact on the preemergent fiber include the following: oxidative metabolism, smoking, ${ }^{42}$ UV radiation, ${ }^{43}$ inflammation from microbial, pollutant, or irritant origins, and oxidized scalp lipids. Sources of oxidative stress with impact on the postemergent fiber include the following: again UVR, and chemical insults from oxidizing hair colorants and pollutants. ${ }^{44}$

Naito et $\mathrm{al}^{45}$ analyzed the effect of the lipid peroxides on hair follicles, and observed that the topical application of linolein hydroperoxides, one of the lipid peroxides, lead to the early onset of the catagen phase in murine hair cycles. Furthermore, they found that lipid peroxides induced apoptosis of hair follicle cells. They also induced apoptosis in human epidermal keratinocytes by upregulating apoptosisrelated genes. These results indicate that lipid peroxides, which can cause free radicals, induce the apoptosis of hair follicle cells, and this is followed by early onset of the catagen phase.

Bahta et $\mathrm{al}^{46}$ cultured dermal papilla cells (DPC) from balding and nonbalding scalp, and demonstrated that balding DPCs grow slower in vitro than nonbalding DPCs. Loss of proliferative capacity of balding DPCs was associated with changes in cell morphology, expression of senescence-associated $\beta$-galactosidase, decreased expression of proliferating cell nuclear antigen and Bmi-1, upregulation of p16(INK4a)/ $\mathrm{pRb}$ and nuclear expression of markers of oxidative stress and DNA damage, including heat shock protein-27, superoxide dismutase catalase, ataxia-telangiectasia-mutated kinase (ATM), and ATM- and Rad3-related protein. The finding of premature senescence of balding DPC in vitro in association with expression of markers of oxidative stress and DNA damage suggests that balding DPCs are particularly sensitive to environmental stress.

Upton et al ${ }^{47}$ further investigated the effects of oxidative stress on balding and occipital scalp DPCs. Patient-matched DPCs from balding and occipital scalp were cultured at atmospheric $(21 \%)$ or physiologically normal (2\%) 02 . At $21 \%$ O2, DPCs showed flattened morphology and a significant reduction in mobility, population doubling, increased levels of reactive oxygen species and senescence-associated $\beta$-Gal activity, and increased expression of p16(INK4a) and pRB. Balding DPCs secreted higher levels of the negative hair growth regulators TGF $\beta 1$ and 2 in response to $\mathrm{H} 2 \mathrm{O} 2$ but not cell culture-associated oxidative stress. Balding DPCs had higher levels of catalase and total glutathione but appeared to be less able to handle oxidative stress compared with occipital DPCs.

These in vitro studies suggest that there may be a role for oxidative stress in the pathogenesis of PHL not only in relation to cell senescence and migration but also secretion of known hair follicle inhibitory factors, offering opportunities for treatment of PHL beyond minoxidil and the 5AR inhibitors at the same time.

\section{Therapeutic Challenges}

The aim of therapy is to retard the progression of hair thinning and increase hair coverage of the scalp. Clinical and investigative advances have helped us to understand some of the pathogenic steps leading to PHL. Besides genetic factors and peculiarities of androgen metabolism, additional pathogenic factors such as microbial flora, endogenous and exogenous stress, microinflammation, and possibly others are suspected. While further suspects are likely to be exposed, individual diversity of causal agents, as well as of the sequence of events, or combined factors, must be kept in mind when addressing the biological conditions contributing to PHL. The large number of therapeutic molecules currently claimed to be active and patented in this field, and their limited efficacy in offering a definitive cure of PHL, confirm that the mechanism of PHL is highly complex.

Currently, two Food and Drugs Administration (FDA)approved drugs are available for this purpose: oral finasteride, at a dose of $1 \mathrm{mg}$ per day, and topical solution of minoxidil. $^{48}$

\section{Finasteride}

Finasteride represented a major breakthrough in the treatment of MPHL, based on an understanding of the underlying pathophysiology and observations on the respective genetic defect of 5AR. Finasteride is a competitive inhibitor of type 2 5AR and inhibits the conversion of testosterone to DHT. So far, clinical studies have demonstrated both a high efficacy of treatment and a very favorable safety profile, establishing the drug as first-line treatment of MPHL in men aged between 18 and 40 years. ${ }^{49}$

More recently, postfinasteride syndrome (PFS) has been claimed to occur in men who have taken oral finasteride to treat hair loss. While the incidence of persistent sexual, mental, and physical side effects despite quitting finasteride is unknown, and the condition is not recognized by the scientific community, individuals who suffer from PFS do present with very distinctive and homogenous symptoms. The concept has emerged from reports of nondermatologists, neuroendocrinological research, case reports, and uncontrolled studies, and it has been scrutinized by hair experts who found that persistent sexual side effects were only documented in low-quality studies with a strong bias selection and a significant nocebo effect. ${ }^{50}$ Ultimately, low-quality 


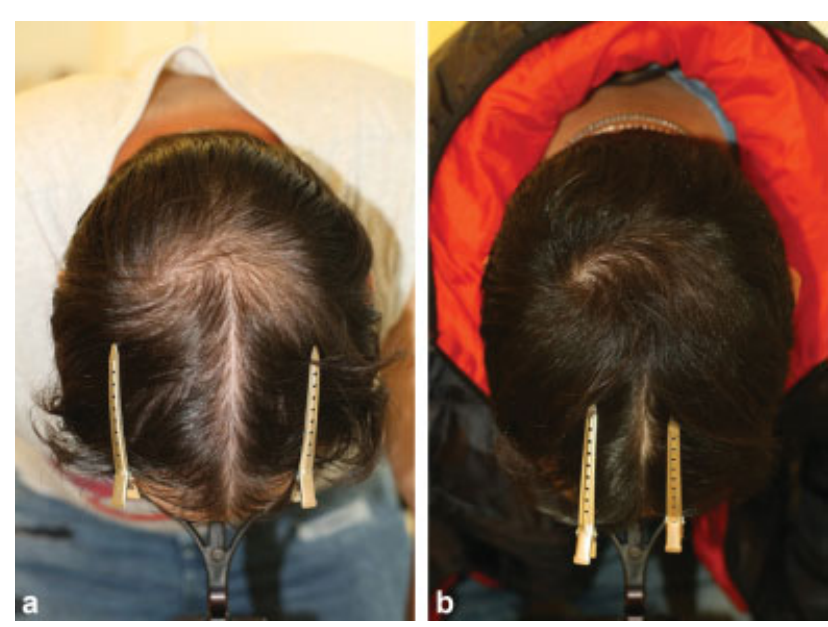

Fig. 1 (a, b) Successful treatment of male pattern hair loss (MPHL) with topical $5 \%$ minoxidil $0.25 \%$ finasteride solution b.i.d (a) before, (b) after 6 months treatment.

studies neither confirm nor refute the condition as a valid nosological entity. Whether the PFS represents a nocebo reaction or a real drug adverse event is irrelevant, while the best way to alleviate the emotional distress related to hair loss is to effectively treat the condition causing the problem. It is not sufficient to only discuss the plausibility of the PFS. ${ }^{51}$ There is a need for practical recommendations to include important issues such as patient selection and risk assessment, appropriate patient information, how to react in case of drug-related adverse events, issues of fertility and malignancy, management of the PFS, and alternatives, specifically the use of topical finasteride..$^{52}$ ( - Fig. 1a, b)

\section{Minoxidil}

Minoxidil promotes hair growth by increasing the duration of anagen. It causes hair follicles at rest to grow and enlarges suboptimal follicles. While minoxidil was developed to treat arterial hypertension, and this feature of the drug's action is best understood, its mechanism of action on hair growth is poorly understood. Several in vitro effects of minoxidil have been described in monocultures of various skin and hair follicle cell types, including stimulation of cell proliferation, inhibition of collagen synthesis, and stimulation of vascular endothelial growth factor and prostaglandin synthesis. Yet, the application of results obtained in cell culture studies to the complex biology of the hair follicle is uncertain. ${ }^{53}$ The discovery of the mutation underlying hypertrichotic osteochondrodysplasia or Cantu's syndrome ${ }^{54}$ has finally shed light on the molecular basis of action of minoxidil. In Cantu's syndrome, the hypertrichosis leads to thick scalp hair extending onto the forehead and a general increase in body hair. It is caused by a mutation in the ABCC9 gene that codes for sulfonylurea receptor 2 , which is involved in ATP-sensitive potassium channels, while minoxidil has been understood to be a potassium-channel opener, with evidence that this effect is mediated by sulfonylurea receptor $2 \mathrm{~B}$. Both the vasorelaxant and hair growth-promoting effect of minoxidil is due to the actions of its sulphated metabolite,

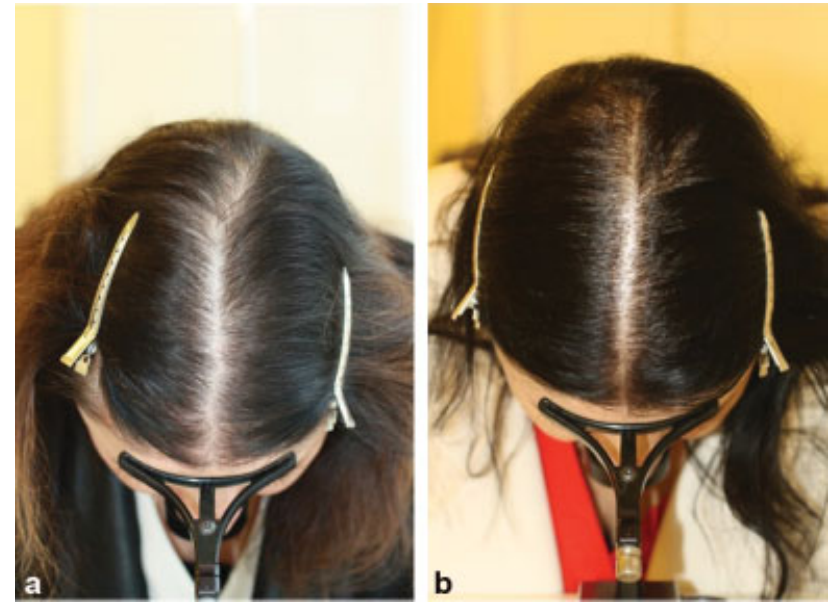

Fig. 2 (a, b) Successful treatment of female pattern hair loss (FPHL) with topical $10 \%$ minoxidil sulfate once daily (a) before, (b) and 3 months after switch from topical 5\% minoxidil b.i.d. to topical 10\% minoxidil sulfate once daily.

minoxidil sulfate. Minoxidil is sulphated by a group of enzymes known as sulfotransferases, some of which are expressed in the hair follicle with wide interindividual variations in the level of enzyme activity. Recent studies have proposed that enzymatic assay of sulfotransferase activity in plucked hair follicles may predict response to topical minoxidil in the treatment of androgenetic alopecia. $^{55}$

Topical solutions of 2 and 5 percent minoxidil are available for treatment of PHL in men and women. In patients with insufficient follicular sulfotransferase activity, $5 \%$ or $10 \%$ minoxidil sulfate solution may be prescribed ( $\mathbf{- F i g}$. $\mathbf{2 a}$, b), or oral minoxidil in a dosage of between 0.625 and $2.5 \mathrm{mg}$ daily, since oral minoxidil is sulphated upon its first pass through the liver (-Fig. 3a, b). Originally proposed by Sinclair, ${ }^{56}$ the efficacy and safety of oral minoxidil for treatment of PHL has been reiterated by others. ${ }^{57}$
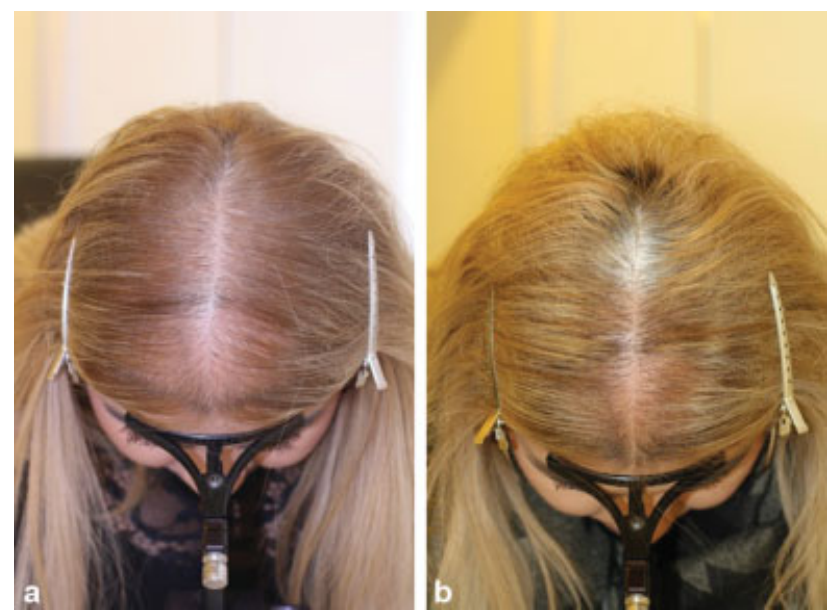

Fig. 3 (a, b) Successful treatment of female pattern hair loss (FPHL) with $1.25 \mathrm{mg}$ oral minoxidil (a) before, (b) after 6 months treatment. 


\section{Hormonal Treatments}

Estrogens and antiandrogens are used in women with FPHL, although no controlled studies have been done. When a combination of estrogen and progestin is prescribed for oral contraception or hormonal replacement therapy in women with FPHL, care should be taken to select a progestin with no androgenic activity.

Topical minoxidil is effective in the treatment of aromatase inhibitor-induced PHL-like alopecia in women treated for breast cancer, providing evidence of the efficacy of minoxidil independent of estrogen levels. ${ }^{58}$

Since Hammerstein's original proposition of the use of antiandrogens to treat women with symptoms of hyperandrogenism, such as hirsutism, seborrhea, and alopecia, ${ }^{59}$ antiandrogen therapy was established as a treatment of FPHL. Typically, a high dosage reverse sequential therapy of $100 \mathrm{mg}$ CPA on the 5th to 14th days of the menstrual cycle and 40 mcg ethinyl estradiol (E2) on the 5th to 25 th days was used in severe cases, while low dosages of $2 \mathrm{mg}$ CPA and 50 mcg of E2 preparations were used for light cases. Spironolactone is yet another agent with antiandrogenic action considered in the treatment of FPHL. Spironolactone is a competitive inhibitor of aldosterone receptors, which also blocks androgen receptors and increases metabolic clearance of testosterone. Finally, flutamide is a nonsteroidal antiandrogen which is used primarily to treat prostate cancer. It is also used off label in the treatment of androgen-dependent conditions in women like acne, excessive hair growth, and alopecia in women.

Meanwhile, the observations of FPHL before pubery, ${ }^{4}$ in patients with hypergonadotropic hypogonadism, ${ }^{14}$ and the androgen-insensitivity syndrome ${ }^{26}$ indicate that the role of androgens may have been overestimated in the pathogenesis of FPHL. Ultimately, Vexiau et al ${ }^{27}$ compared topical minoxidil $2 \%$ with CPA in the treatment of FPHL by randomly assigning 66 women for 12 cycles into two groups, 33 received two local applications ( $2 \mathrm{~mL}$ day 1$)$ of topical minoxidil 2\% plus combined oral contraceptive and 33 received CPA $52 \mathrm{mg}$ day 1 plus E2 35 microg for 20 of every 28 days. The investigators found that minoxidil treatment was more effective in the absence of other signs of hyperandrogenism, hyperseborrhea, and menstrual cycle modifications when the body mass index (BMI) was low, and when nothing argued in favor of biochemical hyperandrogenism, while CPA treatment was more effective when other signs were present and when the BMI was elevated, factors that favored a diagnosis of biochemical hyperandrogenism.

At last, antiandrogen treatment is not without problems. Dose-related side effects of CPA, including weight gain, fatigue, loss of libido, mastodynia, nausea, headaches and depression, are common. Spironolactone may cause breast soreness and menstrual irregularities, and flutamide and related compounds such as bicalutamide may cause fatality due to toxic hepatitis, raising the question of ethical issues related to its use for FPHL while having a questionable rationale and low evidence for efficacy.
Conflict of Interest

None declared.

\section{References}

1 Norwood OT. Male pattern baldness: classification and incidence. South Med J 1975;68(11):1359-1365

2 Norwood OT. Incidence of female androgenetic alopecia (female pattern alopecia). Dermatol Surg 2001;27(01):53-54

3 Venning VA, Dawber RP. Patterned androgenic alopecia in women. J Am Acad Dermatol 1988;18(5 Pt 1):1073-1077

4 Tosti A, Iorizzo M, Piraccini BM. Androgenetic alopecia in children: report of 20 cases. Br J Dermatol 2005;152(03):556-559

5 Trüeb RM. Female pattern baldness in men. J Am Acad Dermatol 1993;29(5 Pt 1):782-783

6 Paus R, Cotsarelis G. The biology of hair follicles. N Engl J Med 1999;341(07):491-497

7 Paus R. Control of the hair cycle and hair diseases as cycling disorders. Curr Opin Dermatol 1996;3:248-258

8 Sawaya ME, Price VH. Different levels of 5alpha-reductase type I and II, aromatase, and androgen receptor in hair follicles of women and men with androgenetic alopecia. J Invest Dermatol 1997;109(03):296-300

9 Randall VA, Thornton MJ, Messenger AG. Cultured dermal papilla cells from androgen-dependent human hair follicles (e.g. beard) contain more androgen receptors than those from non-balding areas of scalp. J Endocrinol 1992;133(01):141-147

10 Itami S, Kurata S, Takayasu S. Androgen induction of follicular epithelial cell growth is mediated via insulin-like growth factor-I from dermal papilla cells. Biochem Biophys Res Commun 1995; 212(03):988-994

11 Hibberts NA, Messenger AG, Randall VA. Dermal papilla cells derived from beard hair follicles secrete more stem cell factor (SCF) in culture than scalp cells or dermal fibroblasts. Biochem Biophys Res Commun 1996;222(02):401-405

12 Kligman AM. The comparative histopathology of male-pattern baldness and senescent baldness. Clin Dermatol 1988;6(04): 108-118

13 Karnik P, Shah S, Dvorkin-Wininger Y, Oshtory S, Mirmirani P. Microarray analysis of androgenetic and senescent alopecia: comparison of gene expression shows two distinct profiles. J Dermatol Sci 2013;72(02):183-186

14 Orme S, Cullen DR, Messenger AG. Diffuse female hair loss: are androgens necessary? Br J Dermatol 1999;141(03):521-523

15 Ellis JA, Stebbing M, Harrap SB. Genetic analysis of male pattern baldness and the 5alpha-reductase genes. J Invest Dermatol 1998; 110(06):849-853

16 Ellis JA, Stebbing M, Harrap SB. Polymorphism of the androgen receptor gene is associated with male pattern baldness. J Invest Dermatol 2001;116(03):452-455

17 Kaufman KD. Androgen metabolism as it affects hair growth in androgenetic alopecia. Dermatol Clin 1996;14(04):697-711

18 Hamilton JB. Male hormone stimulation is prerequisite and an incitant in common baldness. Am J Anat 1942;71:451-480

19 Sawaya ME, Honig LS, Garland LD, Hsia SL. delta 5-3 betahydroxysteroid dehydrogenase activity in sebaceous glands of scalp in male-pattern baldness. J Invest Dermatol 1988;91(02): 101-105

20 Takashima I, Adachi K, Montagna W. Studies of common baldness in the stumptailed macaque. IV. In vitro metabolism of testosterone in the hair follicles. J Invest Dermatol 1970;55(05):329-334

21 Hodgins MB, Murad S, Simpson NB. A search for variation in hair follicle androgen metabolism which might be linked to male pattern baldness. . (abstract)Br J Dermatol 1985;113:794

22 Hoffmann R, Happle R. Current understanding of androgenetic alopecia. Part I: etiopathogenesis. Eur J Dermatol 2000;10(04): 319-327 
23 Trüeb RM, Meyer JC. Male-pattern baldness in men with X-linked recessive ichthyosis. Dermatology 2000;200(03):247-249

24 Imperato-McGinley J, Guerrero L, Gautier T, Peterson RE. Steroid 5alpha-reductase deficiency in man: an inherited form of male pseudohermaphroditism. Science 1974;186(4170):1213-1215

25 Quigley CA. The androgen receptor: physiology and pathophysiology. In: Nieschlag E, Behre HM, eds. Testosterone: Action, Deficiency, Substitution. Berlin: Springer-Verlag; 1998

26 Cousen P, Messenger A. Female pattern hair loss in complete androgen insensitivity syndrome. Br J Dermatol 2010;162(05): $1135-1137$

27 Vexiau P, Chaspoux C, Boudou P, et al. Effects of minoxidil 2\% vs. cyproterone acetate treatment on female androgenetic alopecia: a controlled, 12-month randomized trial. Br J Dermatol 2002;146 (06):992-999

28 Carlini P, Di Cosimo S, Ferretti G, et al. Alopecia in a premenopausal breast cancer woman treated with letrozole and triptorelin. Ann Oncol 2003;14(11):1689-1690

29 Garza LA, Liu Y, Yang Z, et al. Prostaglandin D2 inhibits hair growth and is elevated in bald scalp of men with androgenetic alopecia. Sci Transl Med 2012;4(126):126ra34

30 Chovarda E, Sotiriou E, Lazaridou E, Vakirlis E, Ioannides D. The role of prostaglandins in androgenetic alopecia. Int J Dermatol 2021;60(06):730-735

31 Blume-Peytavi U, Lönnfors S, Hillmann K, Garcia Bartels N. A randomized double-blind placebo-controlled pilot study to assess the efficacy of a 24 -week topical treatment by latanoprost $0.1 \%$ on hair growth and pigmentation in healthy volunteers with androgenetic alopecia. J Am Acad Dermatol 2012;66(05):794-800

32 Jaworsky C, Kligman AM, Murphy GF. Characterization of inflammatory infiltrates in male pattern alopecia: implications for pathogenesis. Br J Dermatol 1992;127(03):239-246

33 Whiting DA. Diagnostic and predictive value of horizontal sections of scalp biopsy specimens in male pattern androgenetic alopecia. J Am Acad Dermatol 1993;28(5 Pt 1):755-763

34 Mahé YF, Michelet JF, Billoni N, et al. Androgenetic alopecia and microinflammation. Int J Dermatol 2000;39(08):576-584

35 Zinkernagel MS, Trüeb RM. Fibrosing alopecia in a pattern distribution: patterned lichen planopilaris or androgenetic alopecia with a lichenoid tissue reaction pattern? Arch Dermatol 2000;136 (02):205-211

36 Reichel M, Heisig P, Kampf G. Identification of variables for aerobic bacterial density at clinically relevant skin sites. J Hosp Infect 2011;78(01):5-10

37 Philpott MP, Sanders DA, Bowen J, Kealey T. Effects of interleukins, colony-stimulating factor and tumour necrosis factor on human hair follicle growth in vitro: a possible role for interleukin- 1 and tumour necrosis factor-alpha in alopecia areata. $\mathrm{Br} \mathrm{J}$ Dermatol 1996;135(06):942-948

38 Lavker RM, Miller S, Wilson C, et al. Hair follicle stem cells: their location, role in hair cycle, and involvement in skin tumor formation. J Invest Dermatol 1993;101(1, Suppl)16S-26S

39 Harman D. Aging: a theory based on free radical and radiation chemistry. J Gerontol 1956;11(03):298-300

40 Arck PC, Overall R, Spatz K, et al. Towards a "free radical theory of graying": melanocyte apoptosis in the aging human hair follicle is an indicator of oxidative stress induced tissue damage. FASEB J 2006;20(09):1567-1569
41 Trüeb RM. The impact of oxidative stress on hair. Int J Cosmet Sci 2015;37(Suppl 2):25-30

42 Trüeb RM. Association between smoking and hair loss: another opportunity for health education against smoking? Dermatology 2003;206(03):189-191

43 Trüeb RM. Is androgenetic alopecia a photoaggravated dermatosis? Dermatology 2003;207(04):343-348

44 Trüeb RM, Henry JP, Davis MG, Schwartz JR. Scalp condition impacts hair growth and retention via oxidative stress. Int J Trichology 2018;10(06):262-270

45 Naito A, Midorikawa T, Yoshino T, Ohdera M. Lipid peroxides induce early onset of catagen phase in murine hair cycles. Int $\mathrm{J} \mathrm{Mol}$ Med 2008;22(06):725-729

46 Bahta AW, Farjo N, Farjo B, Philpott MP. Premature senescence of balding dermal papilla cells in vitro is associated with p16 (INK4a) expression. J Invest Dermatol 2008;128(05): 1088-1094

47 Upton JH, Hannen RF, Bahta AW, Farjo N, Farjo B, Philpott MP. Oxidative stress-associated senescence in dermal papilla cells of men with androgenetic alopecia. J Invest Dermatol 2015;135(05): 1244-1252

48 Price VH. Treatment of hair loss. N Engl J Med 1999;341(13): 964-973

49 Kaufman KD, Olsen EA, Whiting D, et al; Finasteride Male Pattern Hair Loss Study Group. Finasteride in the treatment of men with androgenetic alopecia. J Am Acad Dermatol 1998;39(4 Pt 1):578-589

50 Fertig R, Shapiro J, Bergfeld W, Tosti A. Investigation of the plausibility of 5-alpha-reductase inhibitor syndrome. Skin Appendage Disord 2017;2(3-4):120-129

51 Rezende HD, Dias MFRG, Trüeb RM. A comment on the postfinasteride syndrome. Int J Trichology 2018;10(06): 255-261

52 Suchonwanit P, Srisuwanwattana P, Chalermroj N, Khunkhet S. A randomized, double-blind controlled study of the efficacy and safety of topical solution of $0.25 \%$ finasteride admixed with $3 \%$ minoxidil vs. $3 \%$ minoxidil solution in the treatment of male androgenetic alopecia. J Eur Acad Dermatol Venereol 2018;32 (12):2257-2263

53 Messenger AG, Rundegren J. Minoxidil: mechanisms of action on hair growth. Br J Dermatol 2004;150(02):186-194

54 van Bon BW, Gilissen C, Grange DK, et al. Cantú syndrome is caused by mutations in ABCC9. Am J Hum Genet 2012;90(06): 1094-1101

55 Goren A, Shapiro J, Roberts J, et al. Clinical utility and validity of minoxidil response testing in androgenetic alopecia. Dermatol Ther (Heidelb) 2015;28(01):13-16

56 Sinclair RD. Female pattern hair loss: a pilot study investigating combination therapy with low-dose oral minoxidil and spironolactone. Int J Dermatol 2018;57(01):104-109

57 Randolph M, Tosti A. Oral minoxidil treatment for hair loss: a review of efficacy and safety. J Am Acad Dermatol 2021;84(03): 737-746

58 Trüeb RM. Minoxidil for endocrine therapy-induced alopecia in women with breast cancer-Saint Agatha's blessing? JAMA Dermatol 2018;154(06):656-658

59 Moltz L, Schwartz U, Hammerstein J. [Clinical use of antiandrogens in the female]. Gynakologe 1980;13(01):1-17 